\title{
Renal Pelvis Carcinoma with Renal Vein or Inferior Vena Cava Involvement Linked to Early-onset Lung Metastasis Based on CT Scan Diagnosis
}

\author{
TZU-YAO LIAO ${ }^{1}$, CHUANG-CHI LIAW ${ }^{1}$, KE-HUNG TSUI $^{2}$ and YU-HSIANG JUAN ${ }^{3}$ \\ ${ }^{1}$ Division of Hemato-Oncology, Department of Internal Medicine, ${ }^{2}$ Department of Urology, \\ ${ }^{3}$ Department of Medical Imaging and Intervention, \\ Chang-Gung Memorial Hospital and Chang-Gung University College of Medicine, Taoyuan, Taiwan, R.O.C.
}

\begin{abstract}
Background/Aim: Renal pelvis cancer with invasion of the renal vein or inferior vena cava (IVC) carries a poor prognosis. The present study investigated whether early-onset lung metastasis in these patients contributes to their poor outcome. Patients and Methods: Data were retrospectively collected from hospitalized patients with metastatic renal pelvis urothelial cancer. The parameters used to estimate the risk of lung metastasis were based on computed tomographic $(C T)$ scans. The parameters included sex, age ( $\leq 65$ years or $>65$ years), site (right or left side), metastasis to para-aortic lymph nodes (LNS), suspicion of peritoneal spread, IVC involvement, and renal vein involvement. There were 71 cases including: 40 (56\%) patients with lung metastasis (22 early-onset and 18 lateonset), 68 (96\%) with suspicion of peritoneal spread, 38 (54\%) with para-aortic LN metastasis, 10 (14\%) with IVC involvement, and 53 (74\%) with renal vein involvement. Sixty-four cases were evaluated to estimate the risk of lung metastasis. Results: Tumor involvement in the IVC $(p=0.01)$ and in the renal vein $(p<0.00001)$ were high risk factors for lung metastasis. Conclusion: Tumor involvement of the renal vein or IVC is linked to early-onset lung metastasis in renal pelvis cancer based on CT scan diagnosis.
\end{abstract}

Renal pelvis urothelial cancer with invasion of the renal vein or the inferior vena cava (IVC) has been reported (1-4). Although the incidence of renal vein or IVC venous tumor

Correspondence to: Chuang-Chi Liaw, MD, Division of HematoOncology, Department of Internal Medicine, Chang-Gung Memorial Hospital, 5 Fusing St., Gueishan Township, Taoyuan City, 333, Taiwan, ROC. Tel: +88633281200 ext. 8825, Fax: +886 3278211, e-mail: e102309@adm.cgmh.org.tw

Key Words: Renal pelvis cancer, renal vein, inferior vein cava, lung metastasis, computed tomography. thrombosis is less common than in renal cell carcinoma, the occurrence of thrombosis carries a poor prognosis (1-4).

The renal vein emerges from the renal hilum anterior to the renal artery and drains into the IVC at the level of L2 (5). Renal pelvis cancer can involve either the renal vein or the IVC. Hematogenous spread of tumor cells is the most common path for metastatic lung cancer, with direct venous drainage to the lungs (6). Distal rectal cancers may easily metastasize to the lungs because the inferior rectal vein drains into the IVC (7). When the primary tumor invades the venous system, tumor cells embolize to the lungs through the pulmonary or bronchial arteries (6), and subsequently, tumor nodules can enter the pulmonary veins from tumor metastases (8).

Computed tomography (CT) scans are useful for determining the stage or extent of upper urinary tract cancer (9). We hypothesize that early-onset lung metastasis contributes to the poor prognosis of patients with these carcinomas. The aim of this study, was to investigate the correlation between renal vein or IVC involvement and lung metastasis for renal pelvis carcinomas using CT scans.

\section{Materials and Methods}

Study population. A retrospective case series study was conducted using data collected from patients with metastatic renal pelvis cancer admitted to the oncology ward of Chang-Gung Memorial Hospital, Taoyuan, Taiwan, between January 2010 and December 2017. A single medical oncologist specialized in urological cancer provided most of the data. All patients were hospitalized due to chemotherapy treatment and palliative care for complications. CT scans were performed in all cases in order to evaluate the extent of the tumor.

The link between renal vein or IVC involvement of renal pelvis carcinomas and lung metastasis was evaluated based on the CT scans. The parameters included sex, age ( $\leq 65$ years or $>65$ years), site (right or left side), metastasis to para-aortic lymph nodes (LNs), suspicion of peritoneal spread, IVC involvement, and renal vein involvement. 
Table I. Clinical characteristics of 71 patients with metastatic renal pelvis carcinomas admitted to the oncology ward of Chang-Gung Memorial Hospital, Taoyuan, Taiwan, between January 2010 and December 2017

\begin{tabular}{lc}
\hline Characteristics & No. of patients $(\%)$ \\
\hline Age (years) & \\
Median (range) & $65(39-88)$ \\
Gender & $39 / 32$ \\
Male/Female & \\
Histology/cytology & $67(94)$ \\
Urothelial carcinoma & $3(4)$ \\
Squamous cell carcinoma & $1(1)$ \\
Lympho-epithelial like carcinoma & \\
Lung metastases & $40(56)$ \\
Yes & $24(34)$ \\
No & $7(10)$ \\
Difficult to identify & \\
Suspect peritoneal spread & $68(96)$ \\
Yes & $3(4)$ \\
No & \\
Site & $35(49)$ \\
Right & $36(51)$ \\
Left & \\
Para-aortic LN involvement & $38(54)$ \\
Yes & $33(46)$ \\
No & \\
IVC involvement & $10(14)$ \\
Yes & $61(86)$ \\
No & $53(74)$ \\
Renal vein involvement & $15(21)$ \\
Yes & $3(4)$ \\
No & \\
Difficult to identify & \\
\hline
\end{tabular}

Renal vein involvement was defined as the detection of a tumor invading the renal vein with venous tumor or thrombus on a CT scan. If renal vein involvement was difficult to identify due to a large renal tumor or hydro-nephrosis, it was defined as difficult to identify. Earlyonset of lung metastasis was defined as the occurrence of lung metastases within one month of detecting tumor involvement of the renal vein. Late-onset lung metastasis was defined as the occurrence of lung metastases more than one month after the detection of tumor involvement of the renal vein. Lung metastases were defined as difficult to identify when lung-infiltrating lesions were present but their confirmation as metastatic lung lesions were difficult to be obtained based on their chest films and CT scans. If peritoneal metastasis could not be confirmed, but thick and/or dirty peritoneal lesions were detected on the CT scan, peritoneal spread was suspected.

Statistical analysis. We used Fisher's exact test to detect differences between subgroups, and a $p$-value $<0.05$ was considered statistically significant.

\section{Results}

There were 71 consecutive patients with metastatic renal pelvis cancer, including 39 men and 32 women aged
Table II. Risk factors for lung metastasis evaluated in 64 patients with metastatic renal pelvis carcinoma admitted to the oncology ward of Chang-Gung Memorial Hospital, Taoyuan, Taiwan, between January 2010 and December 2017.

\begin{tabular}{|c|c|c|}
\hline Total cases $(n=64)$ & $\begin{array}{c}\text { Lung metastases } \\
\text { cases }(n=42) / \\
\text { Total cases }(n=64)(\%)\end{array}$ & $p$-Value \\
\hline Age & & 0.07 \\
\hline$>65$ years & $22 / 29(76)$ & \\
\hline$\leq 65$ years & $18 / 35(51)$ & \\
\hline Gender & & 0.30 \\
\hline Male & $23 / 33(70)$ & \\
\hline Female & $17 / 31(55)$ & \\
\hline Site & & 0.31 \\
\hline Right & $16 / 29(55)$ & \\
\hline Left & $24 / 35(71)$ & \\
\hline Para-aortic LN involvement & & 0.31 \\
\hline Yes & 24/35 (71) & \\
\hline No & $16 / 29(55)$ & \\
\hline Suspect peritoneal spread & & 0.08 \\
\hline Yes & $40 / 68(59)$ & \\
\hline No & $0 / 3(0)$ & \\
\hline IVC involvement & & 0.01 \\
\hline Yes & $10 / 10(100)$ & \\
\hline No & $30 / 54(56)$ & \\
\hline Renal vein involvement & & $<0.00001$ \\
\hline Yes & $40 / 28(85)$ & \\
\hline No or Difficult to identify & $0 / 3(0)$ & \\
\hline
\end{tabular}

LN: Lymph node; IVC: inferior vena cava.

between 39 and 88 years (median age, 65 years) enrolled in this study. Of them, 26 patients received nephroureterectomy procedures. Sixteen patients had multiple primary sites of urinary cancer, including six cases involving the bladder (one case simultaneously), and 10 cases involving the ureter (six cases simultaneously). One case had a previous renal transplantation, and one case had end stage renal disease.

The patients' clinical characteristics are shown in Table I. Most common pathology in the study was urothelial carcinoma. Lung metastasis occurred in $40(56 \%)$ patients and was the most common site of metastasis. This included $22(55 \%)$ patients with early-onset lung metastasis and 18 (45\%) patients with late-onset lung metastasis. Other metastatic sites included the liver in 7 , bone metastases or lumbar spine invasion in 18, left supraclavicular LNs in 5, the central nervous system in 3 , and adrenal invasion in 3 . Suspected peritoneal spread was confirmed in $68(96 \%)$ patients. Tumor involvement of para-aortic LNs, IVC, and renal vein was seen in 38 (54\%), $10(14 \%)$, and 53 (74\%) patients, respectively.

Sixty-four cases were evaluated to estimate the risk of lung metastasis (Table II). This excluded the seven cases 

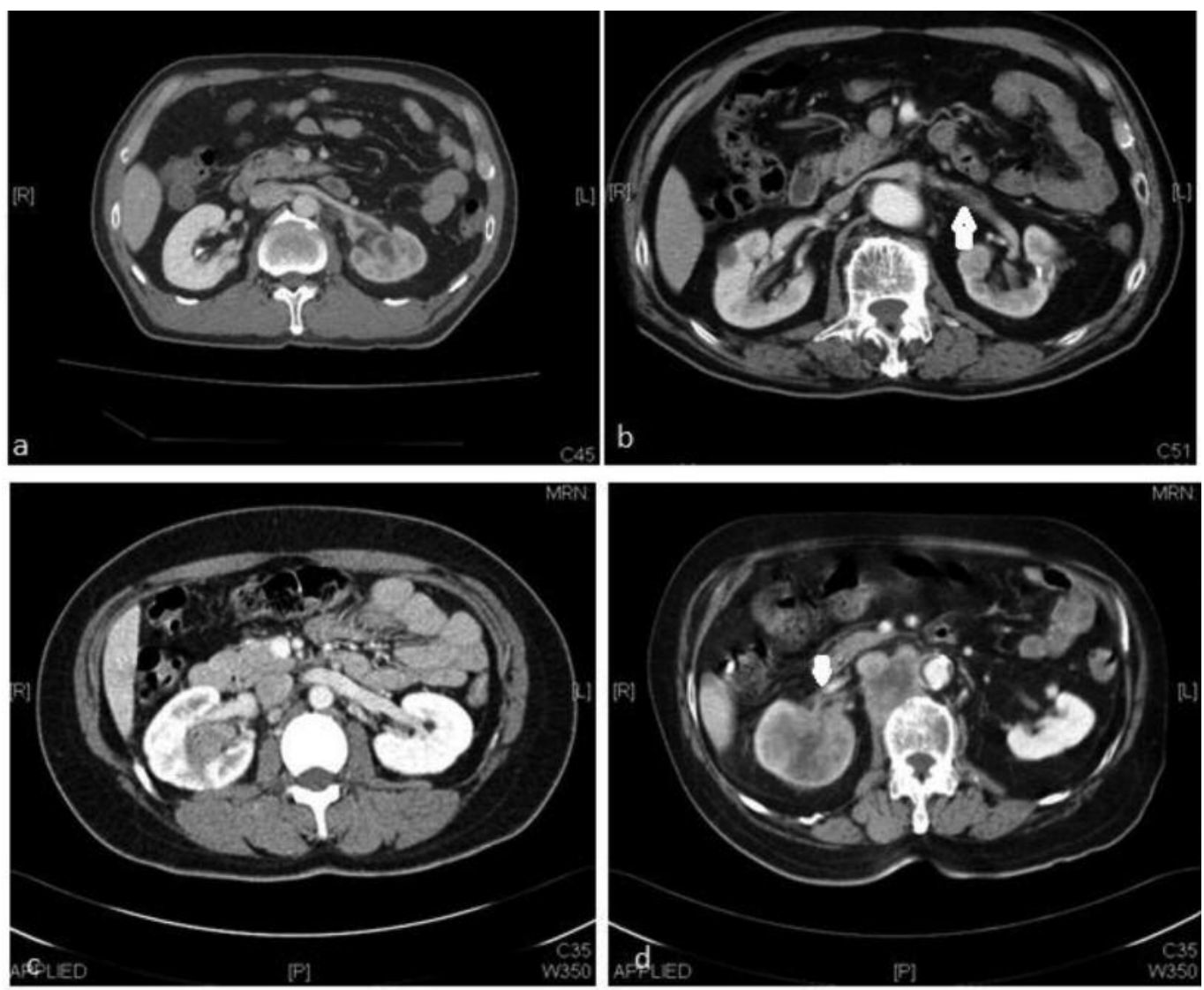

Figure 1. CT scans showing involvement of the renal vein in renal pelvis carcinoma. Left renal pelvis carcinoma with patent left renal vein (A); left renal pelvis carcinoma with tumor invasion of the left renal vein $(B)$; right renal pelvis carcinoma with patent right renal vein $(C)$; and right renal pelvis carcinoma with tumor invasion of the right renal vein $(D)$. Arrows indicate the tumor involving renal veins.

where lung metastasis was difficult to identify. We found that tumor involvement in the IVC $(p=0.01)$ and in the renal vein $(p<0.00001)$ were high risk factors for lung metastasis.

Figure 1 shows CT scans of renal pelvis carcinomas with patent renal veins (Figure $1 \mathrm{~A}$ and $\mathrm{C}$ ) and tumor involvement of the renal vein (Figure 1B and D). Figure 2 shows a CT scan of a case with tumor involvement of the renal vein and lung metastases (Figure 2A and B), and another case with a patent renal vein and no lung metastases (Figure 2C and D). Figure 3 shows a CT scan of a case with tumor involvement of the renal vein and Gerota's fascia (Figure 3A and B) and the presence of lateonset lung metastasis (Figure 3C). Figure 4 shows a CT scan of a case with tumor involvement of the renal vein where the lesion is not located at the proximal segment of the renal vein (Figure 4A and B) and the presence of earlyonset lung metastases (Figure 4C). Figure 5 shows the CT scan of a case with tumor involvement of both the renal vein and the inferior vena cava.

\section{Discussion}

The most common sites of metastasis from renal pelvis cancer are the lymph nodes, bones, lungs, liver, and peritoneum (10). Our study had similar findings, with metastases to lungs and para-aortic lymph nodes seen in more than half of our patients, of which more than half had early-onset lung metastasis. Suspicion of peritoneal spread was detected in $96 \%$ of our patients. Peritoneal spread is probable due to direct spread along peritoneal ligaments (11, 12). The Gerota's fascia passes anterior to the kidney, and a tumor on Gerota's fascia may spread to the peritoneum $(12,13)$. Distal rectal cancer has a tendency for lung metastasis due to drainage of blood from the inferior rectal vein to the internal iliac vein and the IVC (6). This is similar to renal pelvis cancers with drainage of blood from the renal vein to the IVC (5). In our study, tumors involving the renal vein were seen in nearly three quarters of the patients. Tumor invasion of the renal vein may be caused by tumor involvement of Gerota's fascia $(13,14)$. Tumors 


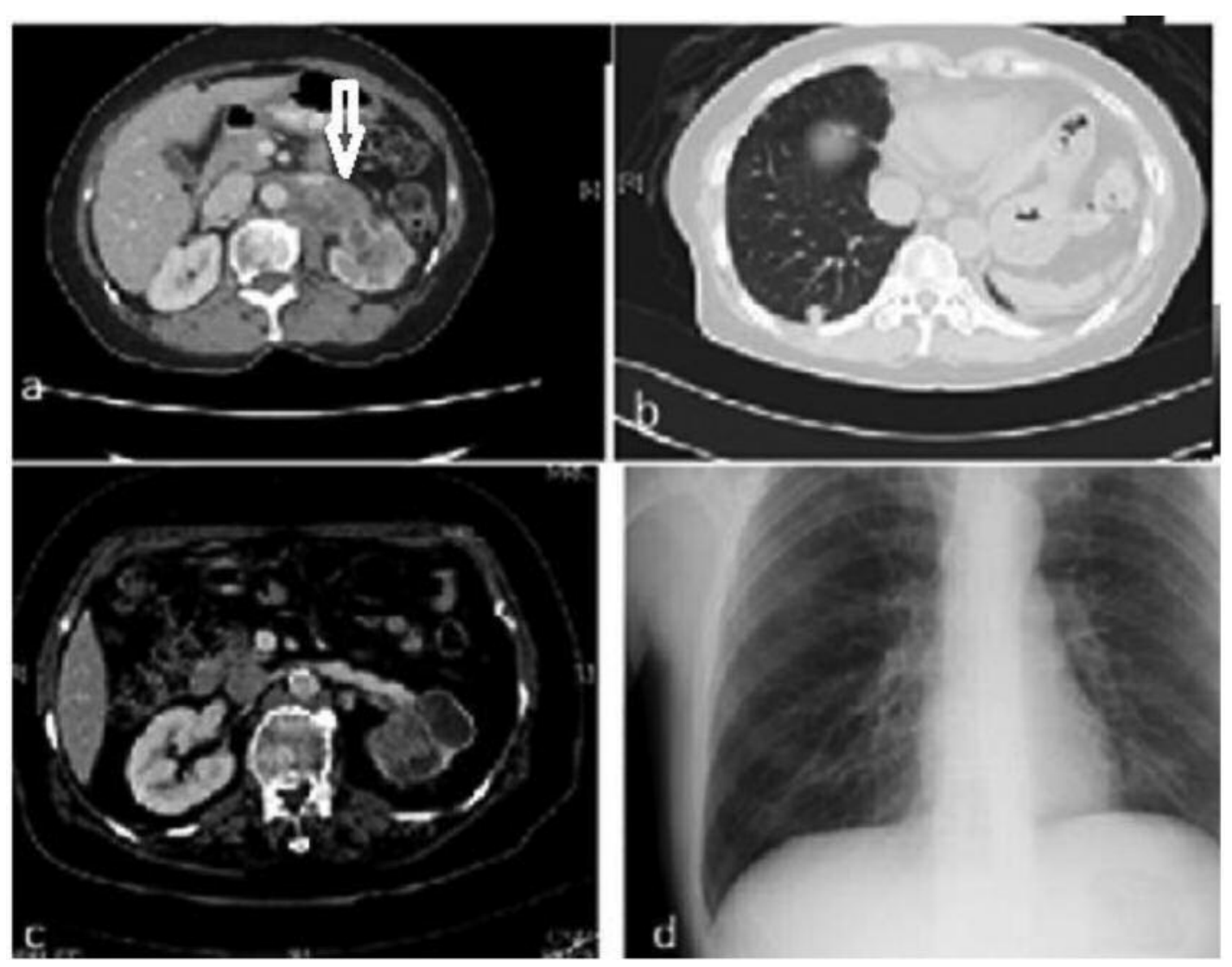

Figure 2. CT scan showing renal pelvis carcinoma with renal vein involvement association with lung metastases. Left renal vein invaded by the tumor and lung metastases with pleural effusion detected $(A, B)$. Patent left renal vein without tumor involvement and chest film showing no presence of lung metastases $(C, D)$. Arrows indicate the tumor involving the renal vein.
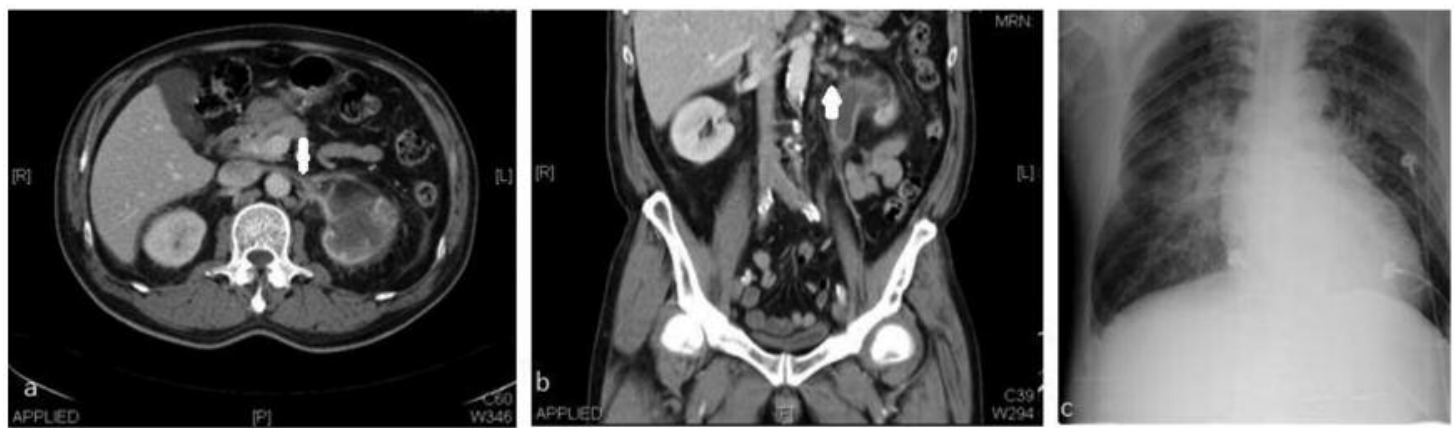

Figure 3. A 63-year-old male with left renal pelvis urothelial carcinoma. CT scan imaging in the axial (A) and coronal (B) views showing tumor invasion of the left renal vein and Gerota's fascia, and a chest plain film showing lung metastases (C). Arrows indicate the tumor involving the renal vein and the arrowhead indicates the tumor involving the Gerota's fascia.

involving the renal veins were not located at the proximal segment of the renal vein. The $\mathrm{T}$ staging system of renal cell carcinoma by the American Joint Committee on Cancer (AJCC), where stage T3b describes a tumor that grossly extends into the renal vein and $\mathrm{T} 4$ describes a tumor that invades beyond Gerota's fascia $(14,15)$. Thickening of Gerota's fascia is considered a reliable sign of locally advanced renal cancer (14). 

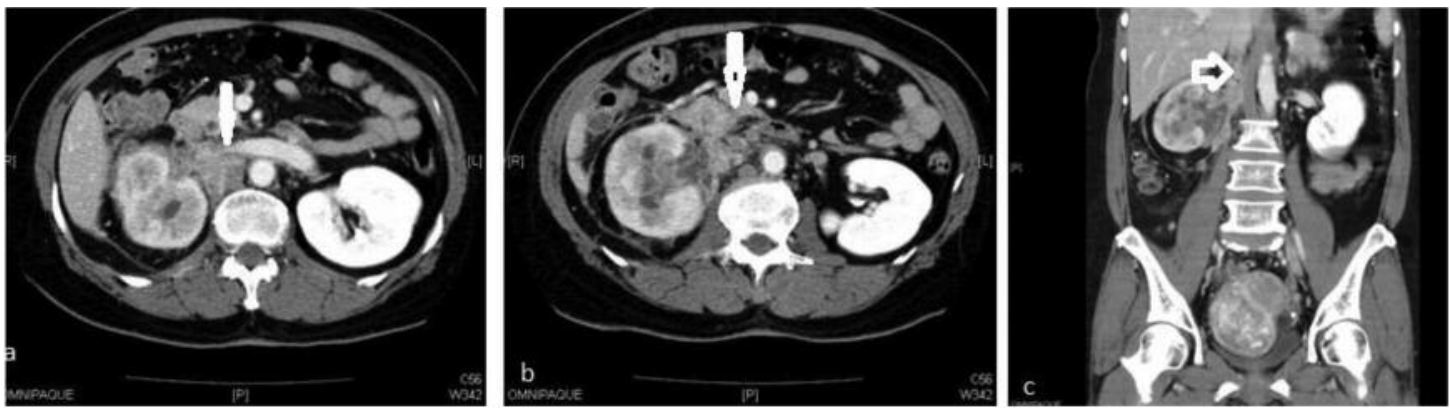

Figure 4. A 74-year-old female with left renal pelvis urothelial carcinoma. CT scan imaging in the axial (A) and coronal (B) views showing tumor invasion of the left renal vein, and a chest plain film showing early lung metastases $(C)$. Arrows indicate the tumor involving the renal vein. Note that the lesion involving the renal vein is not located at the proximal segment of the renal vein.
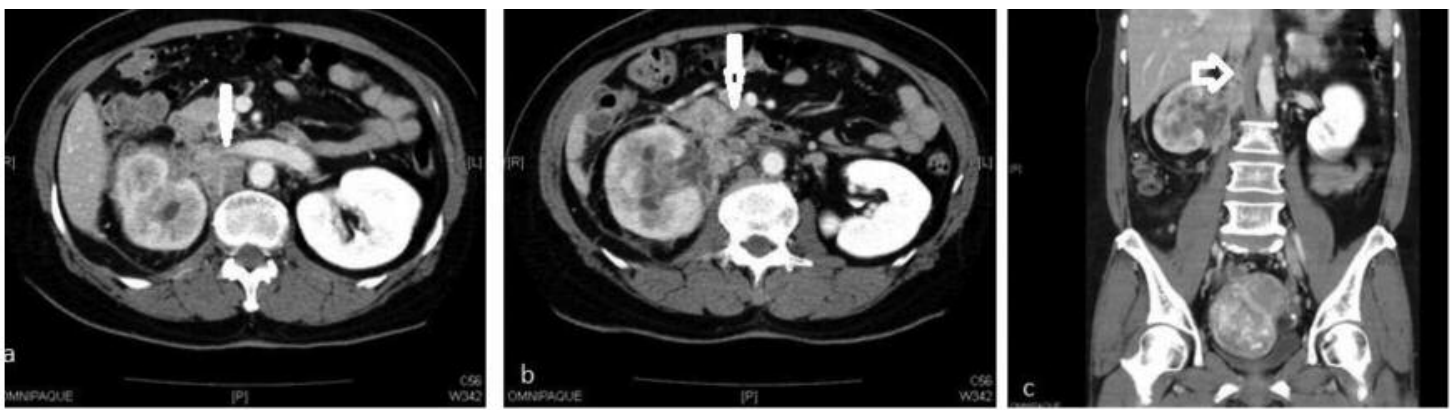

Figure 5. A 57-year-old female with right renal pelvis urothelial carcinoma. CT scan imaging in the axial (A, B) and coronal (C) views showing tumor invasion of the right renal vein and the inferior vena cava. Arrows indicate the tumor involving the renal vein and the inferior vena cava.

Tumor involvement of the renal vein was seen in $74 \%$ of our cases. A tumor involving the Gerota's fascia can also spread anteriorly to the peritoneum $(11,12,13)$. In our study, IVC and renal vein involvement showed a high risk for lung metastasis; however, suspicion of peritoneal spread was not a statistically significant risk for lung metastasis.

There were several limitations in our study. Firstly, the case series data were collected retrospectively. Secondly, the diagnoses of tumor involvement of the renal vein, IVC, Gerota's fascia, and peritoneum were based only on CT scans and not confirmed by Pathology.

\section{Conclusion}

Tumor involvement of the renal vein or IVC is linked to early-onset lung metastasis based on CT scan diagnosis. Tumor involvement of Gerota's fascia likely results in tumor involvement of the adjacent renal vein and spread to the peritoneum.

\section{Conflicts of Interest}

The Authors declare no potential conflicts of interest.

\section{Acknowledgements}

The Authors wish to thank the oncology nursing staff for providing the best possible care for the patients of this study.

\section{References}

1 Singh O, George AJP, Singh JC and Devasia A: Transitional cell carcinoma of the renal pelvis with venous tumor thrombus. Rev Urol 19: 145-148, 2017.

2 Li M, Shi A, Kong W, Zhang J, Chen Y, Huang J and Huang Y: Transitional cell carcinoma with extension of the renal vein and IVC tumor thrombus: report of three cases and literature review. World J Surg Oncol 14: 309, 2016.

3 T Geiger J, Fong Q and Fay R: Transitional cell carcinoma of renal pelvis with invasion of renal vein and thrombosis of subhepatic inferior vena cava. Urol Int 59: 263-265, 1997.

4 Fujimoto M, Tsujimoto Y, Nonomura N, Kojima Y, Miki T, Ariyoshi H, Kawasaki T, Sakon M, Monden M and Okuyama A: Renal pelvic cancer with tumor thrombus in the vena cava inferior. A case report and review of the literature. Urol Int 59: 263-265, 1997.

5 Netter FH: Atlas of Human Anatomy Including Student Consult Interactive Ancillaries and Guides (6th edition). Philadelphia, Penn., W B Saunders Co., pp. 315, 2014. 
6 Pendergrass RCL: Lung metastases; survey of incidence, route of spread, and diagnosis of pulmonary metastases. J Med Assoc Ga 43: 338-341, 1954.

7 Chiang JM , Hsieh PS, Chen JS, Tang R, You JF and Yeh CY: Rectal cancer level significantly affects rates and patterns of distant metastases among rectal cancer patients post curativeintent surgery without neoadjuvant therapy. World J Surg Oncol 2: 197, 2014.

8 Liaw CC, Chang H, Liao TY, Wen MS, Yu CT and Juan YH: The role of pulmonary veins in cancer progression from a computed tomography viewpoint. J Oncol 2016: 1872627, 2016.

9 Buckley JA, Urban BA, Soyer P, Scherrer A and Fishman EK: Transitional cell carcinoma of the renal pelvis: a retrospective look at CT staging with pathologic correlation. Radiology 201: 194-198, 1996.

10 Shinagare AB, Fennessy FM, Ramaiya NH, Jagannathan JP, Taplin ME and Van den Abbeele AD: Urothelial cancers of the upper urinary tract: metastatic pattern and its correlation with tumor histopathology and location. J Comput Assist Tomogr 35: 217-222, 2011
11 Healy JC: Peritoneal metastases. Cancer Imaging 1: 4-12, 2001.

12 Tirkes T, Sandrasegaran K, Patel AA, Hollar MA, Tejada JG, Tann M, Akisik FM and Lappas JC: Peritoneal and retroperitoneal anatomy and its relevance for cross-sectional imaging. Radiographics 32: 437-451, 2012.

13 Amin M, Blandford AT and Polk HCL: Renal fascia of Gerota. Urology 7: 1-3, 1976.

14 Bradley AJ, MacDonald L, Whiteside S, Johnson RJ and Ramani VA: Accuracy of preoperative CT $\mathrm{T}$ staging of renal cell carcinoma: which features predict advanced stage? Clin Radiol 70: 822-829, 2015.

15 Gallardo E, Méndez-Vidal MJ, Pérez-Gracia JL, SepúlvedaSánchez JM, Campayo M, Chirivella-González I, García-DelMuro X, González-Del-Alba A, Grande E and Suárez C: SEOM clinical guideline for treatment of kidney cancer (2017) Clin Transl Oncol 20: 47-56, 2018.

Received March 2, 2018

Revised March 22, 2018

Accepted March 23, 2018 